\title{
The STAT-ROS cycle extends IFN-induced cancer cell apoptosis
}

\author{
YAN WANG*, XIAOYU YU*, HONGTAO SONG, DI FENG, YANG JIANG, SHUANG WU and JINGSHU GENG
}

Department of Pathology, Harbin Medical University Cancer Hospital, Harbin, Heilongjiang 150081, P.R. China

Received July 15, 2017; Accepted October 30, 2017

DOI: 10.3892/ijo.2017.4196

\begin{abstract}
In mammals, the signal transducer and activator of transcription (STAT) protein processes mitochondria importation targets and mitochondria respiratory complexes, and triggers reactive oxygen species (ROS) generation, which conversely rapidly initiates the activation of STAT. Interferon (IFN) administration increases cancer cell apoptosis via STAT activation and ROS accumulation. However, the existence of a STAT-ROS cycle and how it affects IFN-induced cancer cellular apoptosis are unclear. In the present study, we used MCF7 breast cancer cells and confirmed that a combination of IFN- $\alpha / \beta / \gamma$ incubation induced STAT1/3 phosphorylation and mitochondria importation, which increased mitochondria respiratory complexes, the cellular oxygen consumption rate (OCR), and ROS production, followed by cellular apoptosis. We also found that STAT1/3 overexpression induced mitochondria respiratory complexes and ROS production. Additionally, ROS induced by $\mathrm{H}_{2} \mathrm{O}_{2}$ induced phosphorylation of STAT1/3 and promoted mitochondria importation. STAT1/3 deletion suppressed $\mathrm{H}_{2} \mathrm{O}_{2}$-induced acute cellular OCR, increasing the ROS level and indicating that STAT1/3 is necessary for ROS-induced mitochondria OCR and further ROS production, suggesting the existence of a STAT-ROS cycle. We next found that IFN induced mitochondria respiratory complexes followed by induction of OCR, ROS, and apoptosis, which were partially blocked by STAT1/3 deletion. Additionally, the suppression of ROS inhibited IFN-induced STAT1/3 activation. Finally, we discovered that this cycle exists also in A431 and HeLa cancer cells. These results indicate that a STAT-ROS cycle extends IFN-induced cellular apoptosis.
\end{abstract}

\section{Introduction}

The signal transducer and activator of transcription (STAT) proteins, including STAT1-6, share common structural

Correspondence to: Dr Jingshu Geng, Department of Pathology, Harbin Medical University Cancer Hospital, 150 Haping Road, Nangang, Harbin, Heilongjiang 150081, P.R. China

E-mail: jingshugeng@hrbmu.edu.cn

\section{*Contributed equally}

Key words: interferons, STAT1/STAT3, reactive oxygen species, mitochondria, apoptosis, oxygen consumption rate domains of coiled-coil, DNA-binding, linker, $\mathrm{SH} 2$, and transactivation (from $\mathrm{N}$ - to $\mathrm{C}$-terminal), and mediate many aspects of cellular apoptosis, DNA repair, differentiation, and cell cycle progression (1-3). The primary activation of STAT is mediated by Janus kinases (JAK), which transmit extracellular signals to the cytosol and nucleus through transmembrane receptor/ ligands via the JAK-STAT pathway $(4,5)$. In this process, STAT is modified by phosphorylation, glutathionylation, and acetylation, which facilitates STAT activation for transcriptional regulation of target genes (6-8). STAT activation through these modifications contributes to specific cellular responses to cytokines, interleukins, peptide hormones, and growth factors (9-11).

Recently, it was reported that STAT also has a cellular nongenomic function. STAT interacts with GRIM-19, a subunit of mitochondria respiratory complex I, which determines whether STAT3 is imported into mitochondria $(12,13)$. Furthermore, there is direct evidence that STAT is present in the mitochondria of cultured cells and primary tissues. STAT mitochondrial importation selectively stabilizes and increases mitochondria respiratory complexes, allowing them to orchestrate responses to stimuli $(14,15)$. As mitochondria respiratory complex I and III are thought to be the main source for ROS generation $(16,17)$, it has been suggested that STAT1 facilitates ROS production and apoptosis (14). Conversely, STAT activation in some instances seems to depend on ROS signaling $(18,19)$. STAT1 and STAT3 are activated in fibroblasts and A431 carcinoma cells within 5 min after $\mathrm{H}_{2} \mathrm{O}_{2}$ stimulation. Therefore, ROS is thought to be a second messenger to regulate STAT activation (20). These reports strongly indicate that STAT and ROS form a positive STAT-ROS feedback loop, but its details are largely unknown.

Interferons (IFNs) belong to a large group of signal cytokines, which are released from host cells to communicate with other cells to trigger immune system defenses (21), helping to eliminate foreign bodies and malignant cells (22). IFNs are divided into three types: type I, represented by IFN- $\alpha$ and IFN- $\beta$; type II, represented by IFN- $\gamma$; and type III, which was more recently discovered. It is already known that IFN- $\gamma$ induces cellular apoptosis mediated by STAT1 activation (23). IFN- $\beta$ inhibits HepG2 cell viability via phosphorylation of STAT2 (24). Also, an IFN- $\alpha /$ IFN- $\gamma$ co-formulation is involved in the IFN-STAT-pathway and apoptosis in U87MG cells (25). Some of the stimulated apoptosis may be explained by the upregulation of mitochondria respiratory complexes and ROS production $(26,27)$. However, a pilot study of clinical IFN- $\alpha$ administration in melanoma patients indicated that a 
high dose of IFN is not necessary for optimal activation of immune signal transduction (28). This indicates that some latent events beyond the IFN-STAT pathway may be involved in IFN-induced mitochondria respiratory complexes, ROS production, and cellular apoptosis.

In the present study, we directly investigated the potential STAT-ROS cycle in human cancer cells. We found that the STAT-ROS cycle extends the effect of IFN-induced cellular apoptosis. The novel mechanism of the STAT-ROS cycle may be of use to increase the efficacy of IFN administration clinically.

\section{Materials and methods}

Cell culture and reagents. The human breast cancer cell line of MCF7, carcinoma cell line A431, and cervical cancer cell line HeLa (Sigma, Shanghai, China) were cultured in RPMI-1640 (Thermo Fisher Scientific, Shanghai, China) supplemented with $10 \%$ FCS (heat-inactivated), $100 \mathrm{U} / \mathrm{ml}$ penicillin, and $100 \mathrm{mg} / \mathrm{ml}$ streptomycin, and were incubated at $37^{\circ} \mathrm{C}$ in a humidified atmosphere with $5 \% \mathrm{CO}_{2}$.

For some experiments, a combination of recombinant $100 \mathrm{U} / \mathrm{ml}$ IFN- $\alpha 2 \mathrm{a}, 100 \mathrm{U} / \mathrm{ml}$ IFN- $\gamma$ (Sigma), and $100 \mathrm{U} / \mathrm{ml}$ IFN- $\beta$ (Sino Biological, Beijing, China) were used for cell culture according to a previous report (29).

STAT-overexpression in MCF7 cells was established using adenovirus transfection. Human STAT-1 adenovirus (Ad-h-STAT1) and human STAT-3 adenovirus (Ad-h-STAT3) were purchased from Vector Biolabs (Philadelphia, PA, USA). Briefly, the combined adenoviruses were diluted in RPMI-1640 (containing 10\% FCS) and added to the cells at $37^{\circ} \mathrm{C}$ for $24 \mathrm{~h}$. After Ad-STAT1/3 transfection, the media were replaced with serum-free RPMI-1640 media. After 24 h, cells were used for experiments.

STAT knockdown in MCF7 cells was established by transfection with small interfering RNA (siRNA) (Invitrogen, Carlsbad, CA, USA). STAT1 siRNA (siSTAT1): 5'-GCGGAG ACAGCAGAGCGCCUGUAUU-3'; STAT3 siRNA (siSTAT3): 5'-GCCAAUUGUGAUGCUUCCCUGAUUG-3'; and negative control siRNA (siCont) by using Lipofectamine RNAiMAX reagent (Thermo Fisher Scientific) according to the manufacturer's instructions. Twenty-four hours later, cells were used for various experiments. For $\mathrm{H}_{2} \mathrm{O}_{2}$ treatment, $\mathrm{H}_{2} \mathrm{O}_{2}(100 \mu \mathrm{M})$ was applied to MCF7 cells. N-acetyl-cysteine (NAC) $(20 \mathrm{mM})$ (Sigma) was used for ROS inhibition.

Mitochondria isolation and preparation. A large cell culture was prepared for mitochondria isolation with a $15-\mathrm{cm}$ dish being used per sample. After cells were collected, cellular mitochondria extract buffer 1 ( $80 \mathrm{mM}$ sucrose, $10 \mathrm{mM}$ MOPS) was added and cells were homogenized at $1,500 \mathrm{rpm}$ for $2 \mathrm{~min}$. Then cellular mitochondria extract buffer 2 ( $250 \mathrm{mM}$ sucrose, $20 \mathrm{mM}$ MOPS) was added followed by centrifugation at 2,000 rpm at $4^{\circ} \mathrm{C}$ for $10 \mathrm{~min}$. The supernatant was collected and centrifuged again at $15,000 \mathrm{rpm}$ at $4^{\circ} \mathrm{C}$ for $15 \mathrm{~min}$. The precipitate containing mitochondria was then dissolved in buffer 2 for protein quantification. Fifty micrograms of mitochondria were incubated with $50 \mu \mathrm{l}$ proteinase $\mathrm{K}(50 \mu \mathrm{g} / \mathrm{ml})$ to remove nonmitochondrial proteins (30). Then mitochondria were dissolved in cell lysis buffer (CST, Shanghai, China) for western blotting.
Flow cytometry analysis. We performed FACS analysis to detect apoptosis. Cells were cultured in $60-\mathrm{mm}$ dishes and exposed to IFN for $48 \mathrm{~h}$. Cells $\left(2 \times 10^{5}\right.$ cells $\left./ 500 \mu \mathrm{l}\right)$ were labeled fluorescently to detect apoptotic and necrotic cells by adding $50 \mu \mathrm{l}$ binding buffer and $5 \mu \mathrm{l}$ Annexin V-FITC (Pharmingen, San Diego, CA, USA) as well as $2 \mu 1$ of PI (Cedarlane Laboratories, Hornby, Ontario, Canada) to each sample. Samples were incubated at room temperature for $15 \mathrm{~min}$ after gentle mixing. A minimum of 10,000 cells within the gated region were analyzed by flow cytometry (Coulter Epics Altra flow cytometer; Beckman Coulter, Fullerton, CA, USA).

Extracellular flux analysis. The cellular oxygen consumption rate (OCR) and extracellular acidification rate (ECAR) were measured by a Seahorse XF 24 extracellular flux analyzer (Agilent Technologies, Shanghai, China). At the day of measurement, cells were changed to Flux condition medium and incubated at $37^{\circ} \mathrm{C}$ for $1 \mathrm{~h}$. Then cells were measured by baseline OCR and ECAR 3 times. The average values were calculated as the cellular OCR and ECAR. For measurement of cellular OCR response to $\mathrm{H}_{2} \mathrm{O}_{2}, \mathrm{H}_{2} \mathrm{O}_{2}(100 \mu \mathrm{m})$ was injected into wells after the three basic measurements. Measurements were set to continue until the cellular OCR response returned to the baseline.

MitoSOX measurement. Cells were cultured in 96-well dishes. At the day of measurement, cells were washed twice by sterilized PBS, then cells were changed to $5 \mu \mathrm{M}$ MitoSOX (M36008, Invitrogen, Shanghai, China)-containing HBSS medium for another incubation at $37^{\circ} \mathrm{C}$ for $10 \mathrm{~min}$. After three washes by warmed PBS, cells were measured at Ex/Em: 510/580 and protected from light. The blank was set by normal cells without MitoSOX incubation.

TUNEL assay. MCF7 cells were cultured in multi-glass slides. Before cells were prepared for staining, they were washed by sterilized PBS (5 min for three times), fixed by acetone $\left(-30^{\circ} \mathrm{C}\right)$ for $20 \mathrm{~min}$, then washed with TBS. After a 1-h blocking by BSA at room temperature, cells were reacted using a TdT-FragEL DNA Fragmentation Detection kit (Calbiochem, USA) to quantify apoptosis. Counterstaining with fluorescence mounting medium containing DAPI (blue; Thermo Fisher Scientific, Inc., Waltham, MA, USA) was performed to visualize normal nuclei. Slices were observed by use of a fluorescence microscope (Olympus FluoView ${ }^{\mathrm{TM}}$ FV1200).

Western blotting. Western blotting was performed as previously described (31). Briefly, cells were collected in sterilized PBS. Whole cell lysates or isolated mitochondria were dissolved in cell lysis buffer (CST, \#9803). Total protein was adjusted to $1 \mathrm{mg} / \mathrm{ml}$, and $10 \mu \mathrm{l}$ was applied to SDS-PAGE for $90 \mathrm{~min}$, followed by transfer to PVDF membranes for 90 min. Then membranes were blocked in 5\% skim milk for $>1$ h. After 3 washes by TBST, membranes were incubated in specific antibodies at $4^{\circ} \mathrm{C}$ overnight. Membranes were washed by TBST 3 times, followed by incubation in second antibodies at room temperature for $1 \mathrm{~h}$. After washing by TBST 3 times, a FluorChem E (Cell Biosciences, Beijing, China) imaging system was used to visualize the signals. First antibodies: STAT1 (\#9172; CST), pSTAT1 (Tyr701) (\#7649, CST), STAT3 
A

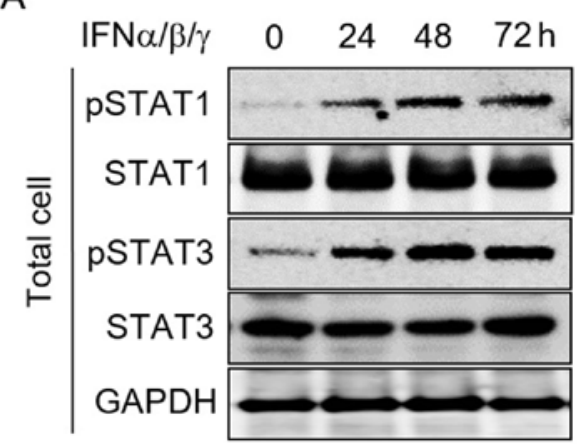

IFN $\alpha / \beta / \gamma \quad 0 \quad 24 \quad 48 \quad 72 \mathrm{~h}$

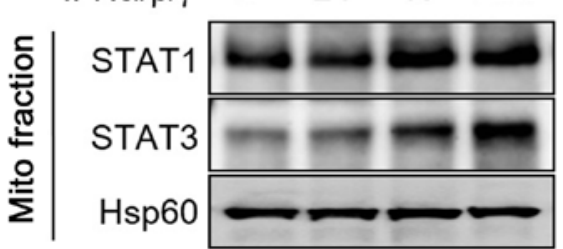

D Cont
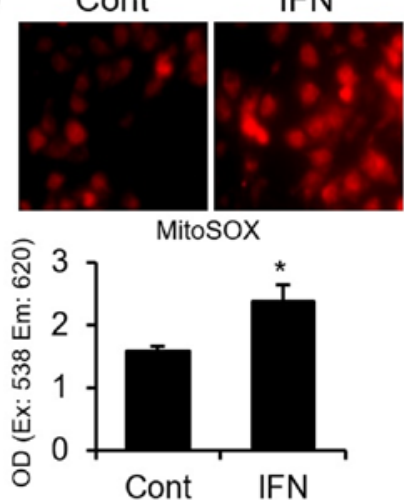

B

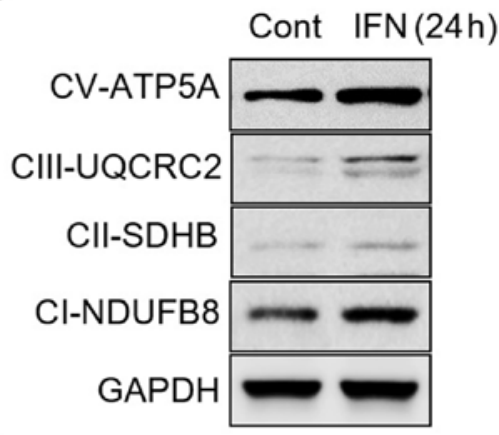

C

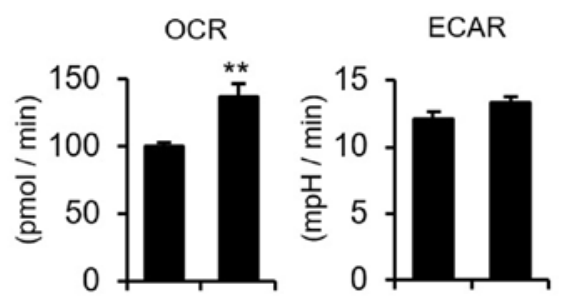

Figure 1. IFN induces STAT1/3 mitochondria importation, and increases OCR, ROS generation, and apoptosis in human MCF7 cells. (A) Representative western blotting of a time-course combination of IFN- $\alpha / \beta / \gamma$-induced cellular and mitochondrial STAT1 and STAT3 activation. GAPDH and HSP60 are loading controls for whole cells and mitochondria subfractions. (B) Representative western blot of mitochondria respiratory complexes after IFN- $\alpha / \beta / \gamma$ incubation. (C) Cellular OCR and ECAR measured at $24 \mathrm{~h}$ after IFN- $\alpha / \beta / \gamma$ incubation ( $\mathrm{n}=10$ for each group). (D) Representative ROS accumulation images of MCF7 cells (upper) and the absorbance measurement of mitoSOX (lower) after IFN incubation ( $\mathrm{n}=8$ for each group). (E) MCF7 cellular apoptosis measured by FACS after IFN incubation. Statistical significance was determined by Student's t-test $\left({ }^{*} \mathrm{p}<0.05 ;{ }^{* *} \mathrm{p}<0.01\right)$.

(\#4904; CST), pSTAT3 (Tyr705) (\#4113; CST), ATP5A (ab110273; Abcam), UQCRC2 (ab103616; Abcam), SDHB (ab14714; Abcam), NDUFB8 (ab192878; Abcam), GAPDH (ab37168; Abcam), Hsp60 (ab46798; Abcam), caspase 3 (\#9662; CST), caspase 9 (\#9508; CST). All first antibodies were diluted 1,000-fold. Second antibodies: anti-mouse IgG antibody (\#7076; CST), anti-rabbit IgG antibody (\#7074; CST). All second antibodies were diluted 2,000-fold.

Statistical analysis. All results are reported as mean values \pm standard error. Comparisons between two groups were performed by unpaired two-tailed t-tests. Multiple comparisons between more than two groups were performed by one-way ANOVA with Tukey's multiple comparisons test between each group. A value of $p<0.05$ was considered significant.

\section{Results}

IFN induces STAT mitochondria importation and increases cellular OCR, ROS, and apoptosis. To clarify the role of IFN in cancer cells, we used MCF7 breast cancer cells. We first performed a time-course study of IFN incubation of MCF7 cells and found that IFN induces STAT1 and STAT3 (STAT1/3) phosphorylation, which reaches a peak after $48 \mathrm{~h}$ of incubation with IFN (Fig. 1A). Meanwhile, after phosphorylation, we found increased STAT1/3 within mitochondria, indicating that IFN induces STAT1/3 mitochondria importation (Fig. 1A). Additionally, IFN increased mitochondria respiratory complexes with upregulated OCR (Fig. 1B and C). Since mitochondria respiratory complexes have been identified as the main source of ROS (16), we examined and detected increased ROS generation induced by IFN (Fig. 1D). The higher ROS thus induced cancer cells apoptosis (Fig. 1E). These results indicated that IFN incubation induces STAT1/3 mitochondria importation, OCR, increased ROS, and subsequent cellular apoptosis.

The feedback between STAT and ROS indicates the existence of a STAT-ROS cycle. Next, we examined the interaction and relationship between STAT and ROS. We overexpressed 

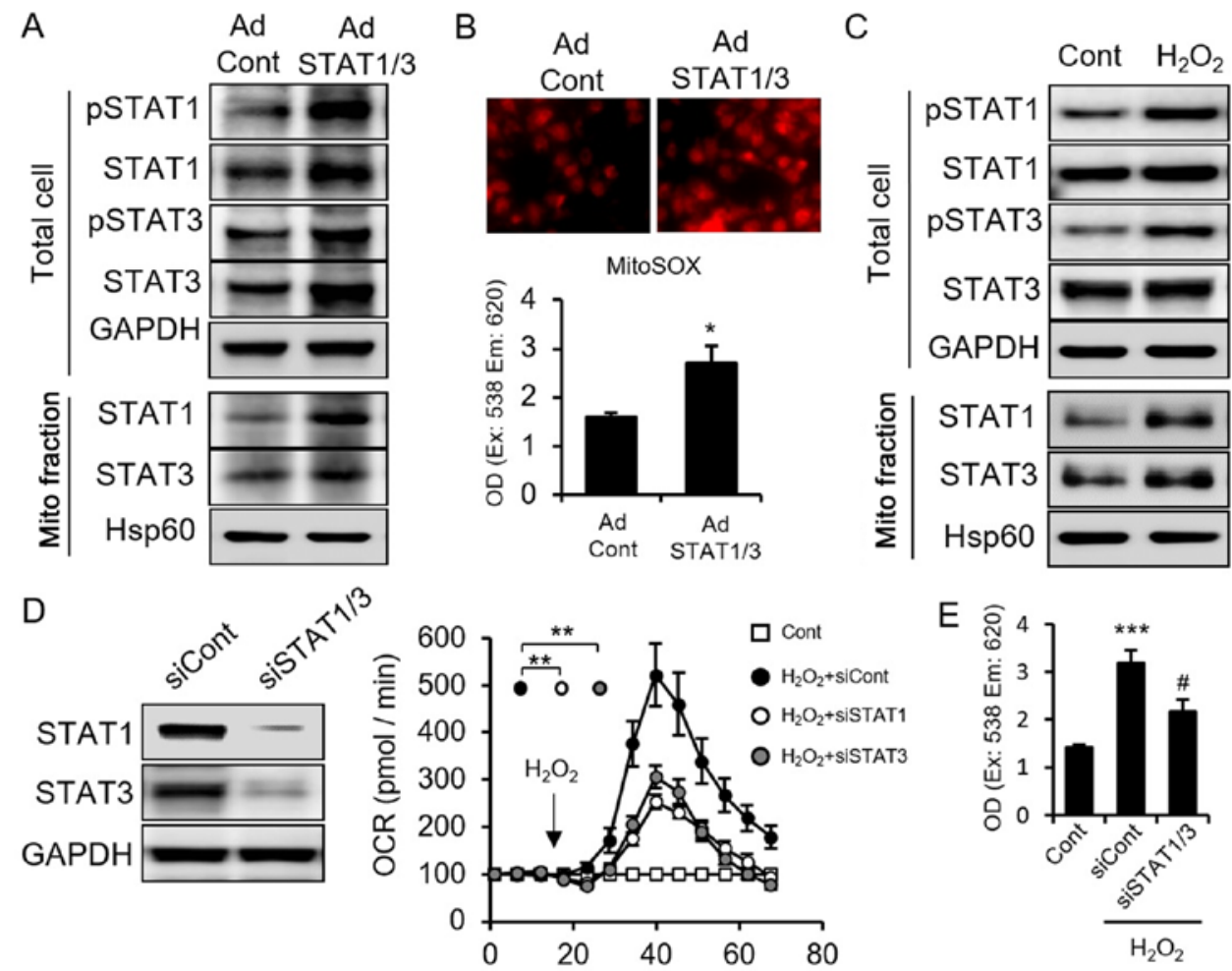

Figure 2. Interaction between STAT and ROS in MCF7 cells. (A) Representative western blot of cellular and mitochondria subfractions showing STAT1 and STAT3 expression as well as phosphorylated fractions after adenovirus-STAT1/3 transfection for $24 \mathrm{~h}$. (B) Representative ROS accumulation images of MCF7 cells (upper) and the absorbance measurement of mitoSOX (lower) after adenovirus-STAT1/3 transfection ( $\mathrm{n}=8$ for each group). (C) Representative western blot of cellular and mitochondria subfractions showing STAT1 and STAT3 expression as well as their phosphorylations after $\mathrm{H}_{2} \mathrm{O}_{2}$ stimulation for $1 \mathrm{~h}$. (D) Representative western blot of STAT1 and STAT3 expression after STAT1/3 siRNA treatment (left). Acute cellular OCR response change after $\mathrm{H}_{2} \mathrm{O}_{2}$ exposure after STAT1/3 siRNA treatment (right) ( $\mathrm{n}=4$ for each group). (E) Absorbance measurement of mitoSOX after STAT1/3 siRNA treatment with or without $\mathrm{H}_{2} \mathrm{O}_{2}$ incubation ( $\mathrm{n}=8$ for each other). Statistical significance was determined by Student's t-test for (B) ( $\mathrm{p}<0.05$ ) and by one-way ANOVA for (E) $\left({ }^{*} \mathrm{p}<0.05\right.$ between Cont and siCont group; ${ }^{*} \mathrm{p}<0.05$ between siCont and siSTAT1/3 group).

STAT1/3 and confirmed their increase in both cellular and mitochondria subfractions (Fig. 2A). Cellular ROS level increased after STAT1/3 overexpression (Fig. 2B), indicating that STAT1/3 induces ROS generation. Conversely, to clarify whether and how STAT1/3 responds to ROS, MCF7 cells were incubated with $\mathrm{H}_{2} \mathrm{O}_{2}$, a type of ROS. We found that $\mathrm{H}_{2} \mathrm{O}_{2}$ increased STAT1 and STAT3 protein expression and induced mitochondria importation (Fig. 2C). The mutual feedback between STAT1/3 and ROS implied the existence of a STAT-ROS cycle.

Recently, it was reported that production of ROS 4-hydroxynonenal, increases OCR, which is accompanied by more ROS production (32). Increased ROS induces more ROS release, known as ROS-induced ROS release (RIRR) (33). So as STAT1/3 and ROS induce each other, we next asked whether STAT1/3 mediates RIRR. We knocked down STAT1/3 using siRNA and measured cellular OCR and ROS levels. Interestingly, knockdown of STAT1/3 suppressed the OCR induced by $\mathrm{H}_{2} \mathrm{O}_{2}$ treatment (Fig. 2D) as well as suppressed $\mathrm{H}_{2} \mathrm{O}_{2}$-stimulated ROS (Fig. 2E). However, we found knockdown of STAT $1 / 3$ did not block the ROS increase induced by $\mathrm{H}_{2} \mathrm{O}_{2}$, that is because $\mathrm{H}_{2} \mathrm{O}_{2}$ induced mitochondrial ROS include two parts: one part is the $\mathrm{H}_{2} \mathrm{O}_{2}$ itself and the another part is the generated ROS from mitochondria stimulated by $\mathrm{H}_{2} \mathrm{O}_{2}$. So, it is correct that STAT1/3 inhibited only parts of the mitochondrial ROS, which maybe the mitochondrial generated ROS by $\mathrm{H}_{2} \mathrm{O}_{2}$. This result also demonstrated STAT1/3 suppression inhibited RIRR. These results suggested that ROS induces OCR, and that ROS generation is partially dependent on STAT $1 / 3$ activation. Taken together, we conclude that STAT $1 / 3$ and ROS form an intercellular STAT-ROS cycle, which amplifies ROS generation and enhances the ROS effect.

The STAT-ROS cycle extends IFN-induced cancer cell apoptosis. As STAT-ROS cycle exists in MCF7 cancer cells (Fig. 2), and IFN induces both STAT1/3 activation and ROS production followed by cellular apoptosis (Fig. 1), we hypothesized that the STAT-ROS cycle plays a positive role in IFN-induced cellular apoptosis. Therefore, we checked whether and how the STAT-ROS cycle affects IFN-induced cellular apoptosis.

First, we confirmed the central role of STAT in IFN-induced cellular apoptosis. STAT1/3 deletion inhibited the IFN incubation-induced increase of mitochondria respiratory complex I and III (Fig. 3A) and the consequent increase in OCR and ROS (Fig. 3B and C). Next, to test whether STAT1/3 deletion could suppress IFN-induced cellular apoptosis, we performed FACS, TUNEL assays, and looked for caspase activation. We found that cellular apoptosis was significantly inhibited by STAT1/3 deletion (Fig. 3D-F), indicating a crucial role of STAT in IFN-induced ROS-dependent cellular apoptosis. Next, to clarify the role of ROS in the STAT-ROS cycle, we checked STAT1/3 activation after ROS inhibition under IFN incubation. We found that ROS inhibition by NAC did not affect the activation of STAT after a relatively short time (24 h) 
A

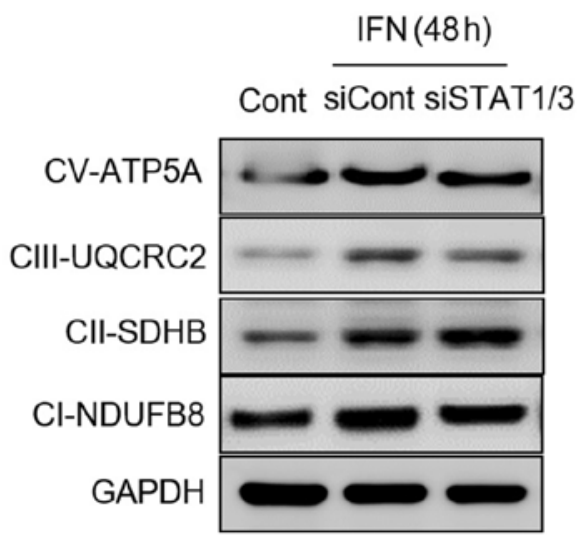

C

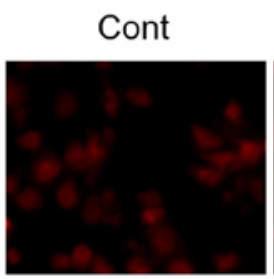

D
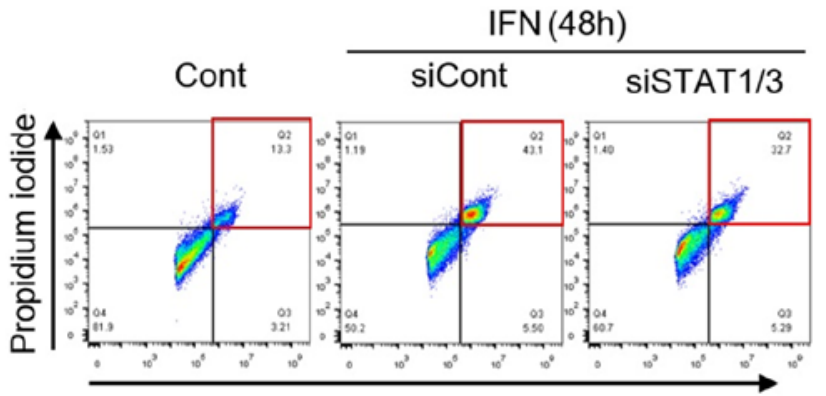

Annexin V-FITC

E

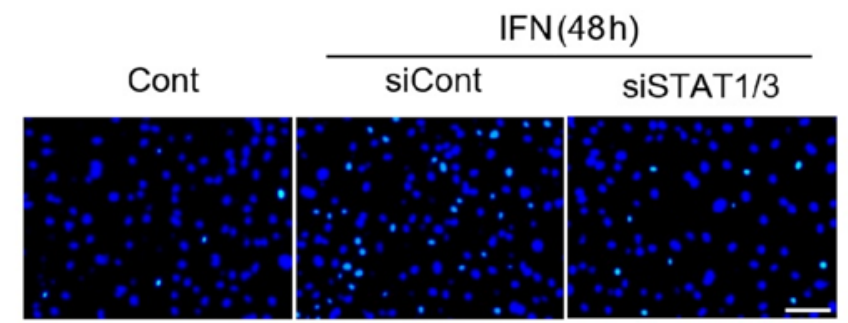

B
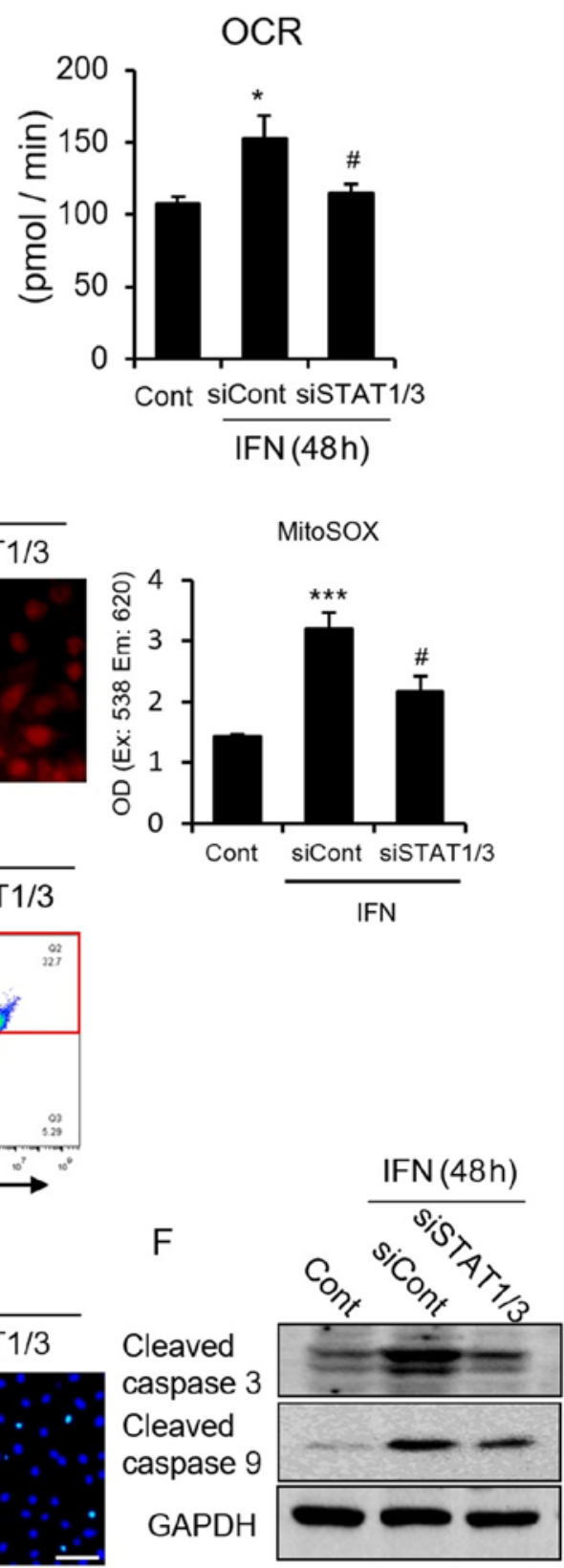

Figure 3. STAT extends the effect of IFN treatment in MCF7 cells. (A) Representative western blot of mitochondria respiratory complexes with or without IFN- $\alpha / \beta / \gamma$ incubation in STAT-knockdown MCF7 cells. (B) Cellular OCR after IFN- $\alpha / \beta / \gamma$ incubation in STAT-knockdown MCF7 cells ( $\mathrm{n}=6$ per each group). (C) Representative ROS accumulation images (upper) and the absorbance measurement of mitoSOX (lower) after IFN- $\alpha / \beta / \gamma$ incubation in STAT1/3-knockdown MCF7 cells ( $\mathrm{n}=8$ for each group). (D and E) MCF7 cellular apoptosis measured by FACS (D) and TUNEL assay (E) after IFN- $\alpha / \beta / \gamma$ incubation in STAT1/3knockdown MCF7 cells. (F) Representative western blot of cleaved caspase 3 and 9 with or without IFN- $\alpha / \beta / \gamma$ incubation in STAT-knockdown MCF7 cells. Statistical significance was determined by one-way ANOVA for (B) (" $\mathrm{p}<0.05$ between Cont and siCont group; ${ }^{*} \mathrm{p}<0.05$ between siCont and siSTAT1/3 group).

during IFN incubation; however, significantly suppressed activation of STAT did occur after a relatively long time $(72 \mathrm{~h})$ (Fig. 4A and B). Taken together, these results suggested that IFN-induced ROS generation is initially dependent on STAT activation, which induces ROS further and activated STAT in a later phase of IFN incubation, indicating that the STAT-ROS cycle extends the effect of IFN on cancer cells.

The STAT-ROS cycle exists in A431 and HeLa cells. We demonstrated that the STAT-ROS cycle extends the effect of IFN-induced apoptosis in MCF7 cells. It has been reported that IFN activates STAT1 and induces apoptosis in both A431 and HeLa cells (34). To determine whether this cycle is common within cancer cells, we examined the STAT-ROS cycle in A431 and HeLa cells. As expected, STAT1/3 deletion in both cell lines partially blocked the $\mathrm{H}_{2} \mathrm{O}_{2}$-induced increase in cellular OCR (Fig. 5A) as well as the $\mathrm{H}_{2} \mathrm{O}_{2}$-induced increase in ROS (Fig. 5B), which were similar to that seen used in the MCF7 cells (Fig. 2D and E). These results indicated STAT suppression inhibited $\mathrm{H}_{2} \mathrm{O}_{2}$ induced mitochondrial RIRR, demonstrated the STAT-ROS cycle exists also in A431 and HeLa cells. 
A

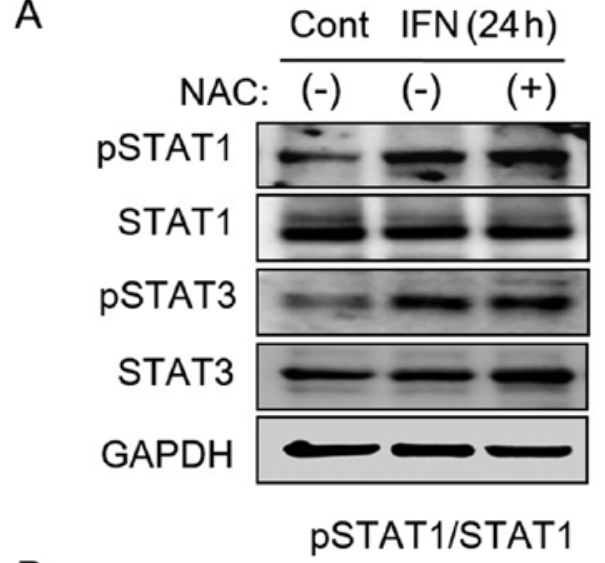

B

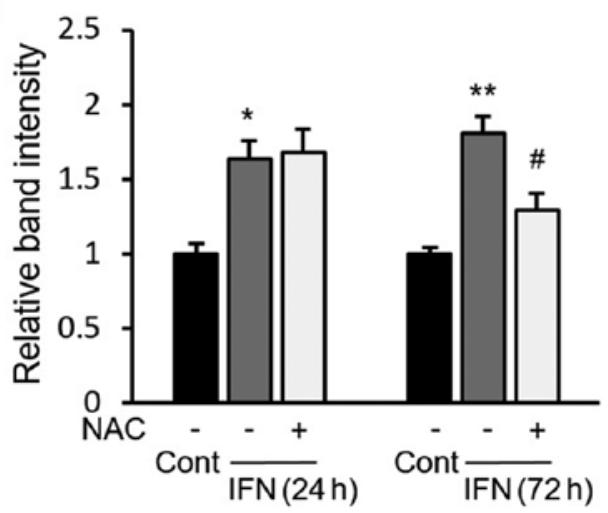

Cont IFN $(72 \mathrm{~h})$
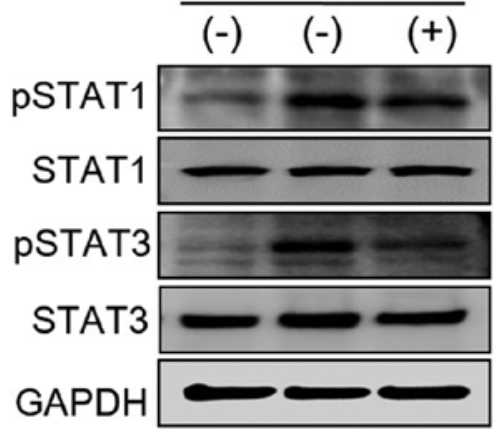

pSTAT3/STAT3

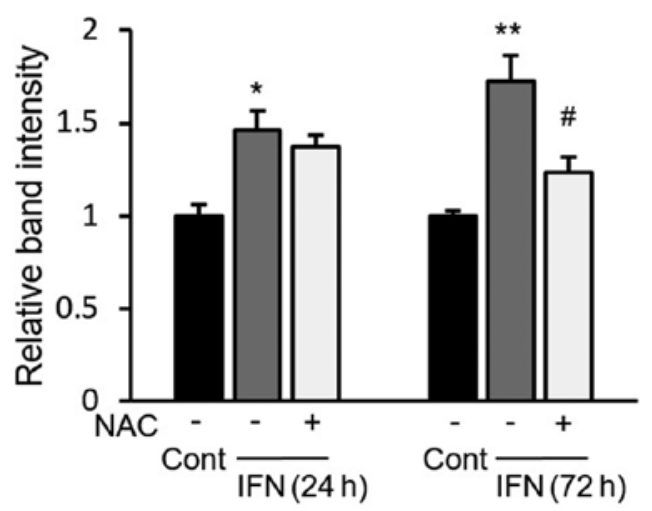

Figure 4. ROS extends the effect of IFN treatment in MCF7 cells. Representative western blot (A) and quantification (B) of STAT1/3 and phosphorylation with or without NAC (ROS inhibitor) after IFN- $\alpha / \beta / \gamma$ incubation for 24 and $72 \mathrm{~h}\left(\mathrm{n}=4\right.$ for each group). ${ }^{*} \mathrm{p}<0.05,{ }^{* *} \mathrm{p}<0.01$ between NAC; $;{ }^{*} \mathrm{p}<0.05$ between $\mathrm{NAC}^{-}$and $\mathrm{NAC}^{+}$after IFN incubation.

A
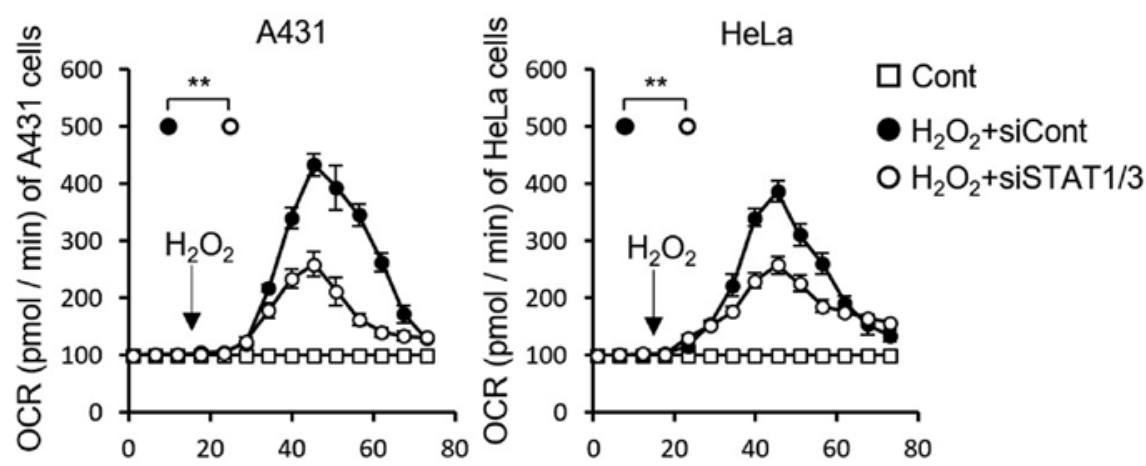

B

A431

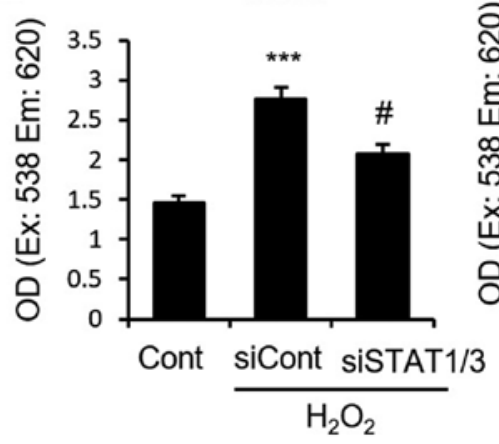

HeLa

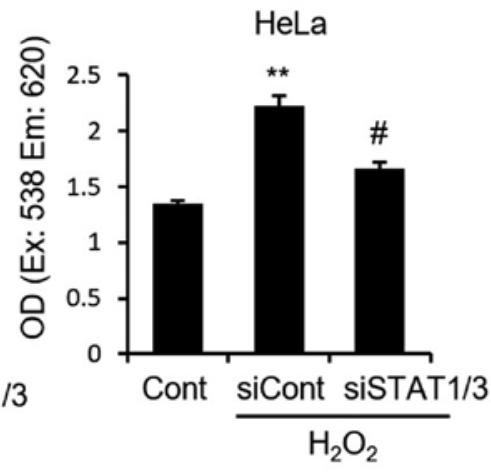

Figure 5. STAT/ROS cycle is observed in A431 and HeLa cells. (A) Acute cellular OCR response change after $\mathrm{H}_{2} \mathrm{O}_{2}$ exposure after STAT1/3 siRNA treatment in A431 cells (left) and HeLa cells (right) ( $\mathrm{n}=4$ for each group). (B) Absorbance measurement of mitoSOX after STAT1/3 siRNA treatment with or without $\mathrm{H}_{2} \mathrm{O}_{2}$ incubation in A431 cells (left) and HeLa cells (right) ( $\mathrm{n}=8$ for each other). ${ }^{* *} \mathrm{p}<0.01,{ }^{* * *} \mathrm{p}<0.005$ between Cont and siCont group; ${ }^{* *} \mathrm{p}<0.01$, ${ }^{\#} \mathrm{p}<0.05$ between siCont and siSTAT1/3 group. 


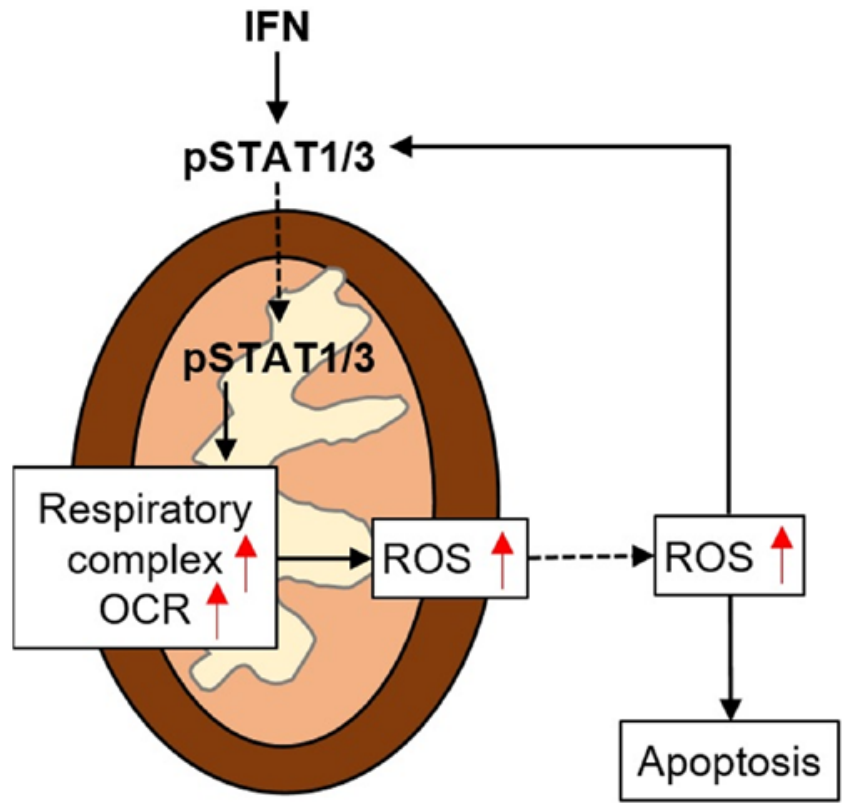

Figure 6. Conceptual schematic of hypothesis that IFNs induce STAT1/3 phosphorylation and subsequent mitochondrial translocation, which induces mitochondrial respiratory complexes and ROS production and release, which in turn further phosphorylates STAT1/3 and induces cellular apoptosis.

\section{Discussion}

The present study demonstrated a potential cycle between STAT1/3 and ROS in MCF7 cancer cells. This STAT-ROS cycle facilitates RIRR to enhance the ROS effect. When STAT1/3 was exposed to IFN, this cycle extended IFN-induced mitochondria-dependent cellular apoptosis. Additionally, we also found that this cycle exists in A431 and HeLa cells, indicating it is a common mechanism in cancer cells to amplify IFN-induced cellular apoptosis (Fig. 6).

Chemical cycles are very important for the efficient usage of reagents and exist universally in vivo. For example, the tricarboxylic acid and lactic acid cycles. Although simpler than these classic chemical cycles, the STAT-ROS cycle may commonly exist in cancer cells, allowing for efficient use of ROS for further ROS production to damage cancer cells.

ROS-STAT cycle push ROS activity, make ROS more efficient than without STAT. Although we have domenstrated this mechanism was involved only in some cancer cells in the present study, we think this cycle seems not only limited to these cancer cells but also commonly exists in some normal cell (these speculation should to be confirmed). So, in our opinion, a bit ROS can be enlarged by ROS-STAT cycle, which makes ROS generationg and release more efficient. Clearly, oxidative stress clearance system was also involved in this cycle. Unquestionably, ROS-STAT cycle enhances the ROS clearance system, which was suppressed by STAT knockdown (data not shown). However, we think the activated ROS clearance system was dependent on ROS level due to ROS-STAT cycle, but not directly affected by this cycle, because ROS could activate oxidative stress clearance system directly.

We found that communication between mitochondria and cytoplasm was necessary for this reactive system. The STAT-ROS cycle begins with exposure to IFN to induce
STAT, but ends with ROS production. It is thus necessary for ROS to move from mitochondria to the cytosol, which requires a reversible mitochondria permeability transition pore (mPTP) opening, an inner membrane anion channel (IMAC) opening, or mitochondria membrane potential $\left(\Delta \psi_{\mathrm{m}}\right)$ loss (33). Interestingly, STAT3 can induce mPTP openings $(35,36)$, and STAT1 and IRF1 synergistically induce $\Delta \psi_{\mathrm{m}}$ loss (14), suggesting that STAT not only induces ROS production but also creates the necessary conditions (mPTP or lost $\Delta \psi_{\mathrm{m}}$ ) to facilitate ROS release from mitochondria. Released ROS induces cellular apoptosis but also further promotes ROS production through STAT mitochondria importation. NFאB has been reported to be activated by ROS and further induces STAT, indicating that NFאB may be involved in ROS-activated STAT (37). However, in addition to STAT1 and STAT3, released ROS also activates STAT5 and STAT6 $(38,39)$, though their effect on mitochondria is largely unknown. Therefore, it is necessary to investigate whether STAT5 or STAT6 are also involved in mitochondria importation.

There are two pathways for STAT signaling, the classical pathway in which phosphorylated STAT is translocated to the nucleus, and the non-classical pathway in which phosphorylated STAT is translocated to mitochondria (40). Interestingly, these two distinct pathways are linked into an integrated system as STAT, in the classical pathway, it induces the transcription of mitochondria genes, while in the non-classical pathway, STAT-mitochondria importation further promotes mitochondrial activities such as ATP production and ROS generation (40). Although the two distinct activities of phosphorylated STAT lead to some loss of mitochondria importation, nucleustranslocated STAT supports mitochondria biogenesis and activity to promote the STAT-ROS cycle.

STAT activation in cancer cells is common (41-47). STAT activates the autonomous proliferation of SUM-102PT and MDA468 human breast cancer cells through an autocrine/ paracrine interaction with $\mathrm{HB}-\mathrm{EGF}$ (41), and in pancreatic cancer cells activates BxPC-3, AsPC-1, Capan-2, MiaPaCa-2, Panc-1, and HPDE-6 $(42,43)$. It is activated in myeloma and lung cancer cells (44) as well as in prostate cancer cells $(45,46)$. Although in most of these cancer cells, STAT phosphorylation is involved in tumor growth, and thus suppression of STAT activation should facilitate cancer cells apoptosis, the STAT/ROS cycle should extend the effect of ROS and facilitate tumor apoptosis $(14,18,19)$. The role of STAT in both tumorigenesis and tumor suppression may be explained by differences in the classical and non-classical pathways (48), namely genomic vs. non-genomic effects. It is therefore very important to understand how STAT classical or genomic pathway and non-classical or non-genomic pathway are mediated or balanced in specific cells. We hypothesize that the non-classical pathway, and not the classical pathway, is involved in STAT activation to induce cancer cell apoptosis. Considering this, the STAT/ROS cycle should only exist in mitochondria or be confined to specific intracellular locations.

In conclusion, we demonstrated the existence of a STAT/ROS cycle in some cancer cells (MCF7, A431 and HeLa). The STAT/ROS cycle involves STAT phosphorylation with mitochondria importation, mitochondria respiratory complex increase, and ROS production and release from mitochondria. This cycle extends the effect of IFN and facilitates 
IFN-induced cancer cell apoptosis. This novel concept may provide new methods for improving IFN therapy.

\section{Acknowledgements}

We thank our colleagues for useful suggestions and comments. This study was supported by a grant from the National Natural Science Foundation of China (81372785).

\section{References}

1. Arbuthnot P, Capovilla A and Kew M: Putative role of hepatitis B virus $X$ protein in hepatocarcinogenesis: Effects on apoptosis, DNA repair, mitogen-activated protein kinase and JAK/STAT pathways. J Gastroenterol Hepatol 15: 357-368, 2000.

2. Beebe K, Lee WC and Micchelli CA: JAK/STAT signaling coordinates stem cell proliferation and multilineage differentiation in the Drosophila intestinal stem cell lineage. Dev Biol 338: 28-37, 2010.

3. Steelman LS, Pohnert SC, Shelton JG, Franklin RA, Bertrand FE and McCubrey JA: JAK/STAT, Raf/MEK/ERK, PI3K/Akt and BCR-ABL in cell cycle progression and leukemogenesis. Leukemia 18: 189-218, 2004.

4. Aaronson DS and Horvath CM: A road map for those who don't know JAK-STAT. Science 296: 1653-1655, 2002.

5. Schindler C and Darnell JE Jr: Transcriptional responses to polypeptide ligands: The JAK-STAT pathway. Annu Rev Biochem 64: 621-651, 1995

6. Yuan ZL, Guan YJ, Chatterjee D and Chin YE: Stat3 dimerization regulated by reversible acetylation of a single lysine residue. Science 307: 269-273, 2005.

7. Xie Y, Kole S, Precht P, Pazin MJ and Bernier M: S-glutathionylation impairs signal transducer and activator of transcription 3 activation and signaling. Endocrinology 150: $1122-1131,2009$

8. Ray S, Boldogh I and Brasier AR: STAT3 NH2-terminal acetylation is activated by the hepatic acute-phase response and required for IL-6 induction of angiotensinogen. Gastroenterology 129: 1616-1632, 2005.

9. Horvath CM: The Jak-STAT pathway stimulated by interleukin 6 . Sci STKE 2004: tr9, 2004.

10. Leonard WJ: Role of Jak kinases and STATs in cytokine signal transduction. Int J Hematol 73: 271-277, 2001.

11. Levy JB, Schindler C, Raz R, Levy DE, Baron R and Horowitz MC: Activation of the JAK-STAT signal transduction pathway by oncostatin-M cultured human and mouse osteoblastic cells Endocrinology 137: 1159-1165, 1996.

12. Lufei C, Ma J, Huang G, Zhang T, Novotny-Diermayr V, Ong CT and Cao X: GRIM-19, a death-regulatory gene product, suppresses Stat 3 activity via functional interaction. EMBO J 22: $1325-1335,2003$.

13. Tamminen P, Anugula C, Mohammed F, Anjaneyulu M, Larner AC and Sepuri NB: The import of the transcription factor STAT3 into mitochondria depends on GRIM-19, a component of the electron transport chain. J Biol Chem 288: 4723-4732, 2013.

14. Lee HJ, Oh YK, Rhee M, Lim JY, Hwang JY, Park YS, Kwon Y, Choi KH, Jo I, Park SI, et al: The role of STAT1/IRF-1 on synergistic ROS production and loss of mitochondrial transmembrane potential during hepatic cell death induced by LPS/d-GalN. J Mol Biol 369: 967-984, 2007.

15. Wegrzyn J, Potla R, Chwae YJ, Sepuri NB, Zhang Q, Koeck T, Derecka M, Szczepanek K, Szelag M, Gornicka A, et al: Function of mitochondrial Stat 3 in cellular respiration. Science 323: 793-797, 2009

16. Chen Q, Vazquez EJ, Moghaddas S, Hoppel CL and Lesnefsky EJ: Production of reactive oxygen species by mitochondria: Central role of complex III. J Biol Chem 278: 36027-36031, 2003.

17. Batandier C, Guigas B, Detaille D, El-Mir MY, Fontaine E, Rigoulet $\mathrm{M}$ and Leverve XM: The ROS production induced by a reverse-electron flux at respiratory-chain complex 1 is hampered by metformin. J Bioenerg Biomembr 38: 33-42, 2006.

18. Kim HS, Cho IH, Kim JE, Shin YJ, Jeon JH, Kim Y, Yang YM, Lee KH, Lee JW, Lee WJ, et al: Ethyl pyruvate has an antiinflammatory effect by inhibiting ROS-dependent STAT signaling in activated microglia. Free Radic Biol Med 45: 950-963, 2008.
19. Liu T, Castro S, Brasier AR, Jamaluddin M, Garofalo RP and Casola A: Reactive oxygen species mediate virus-induced STAT activation: Role of tyrosine phosphatases. J Biol Chem 279: 2461-2469, 2004.

20. Simon AR, Rai U, Fanburg BL and Cochran BH: Activation of the JAK-STAT pathway by reactive oxygen species. Am J Physiol 275: C1640-C1652, 1998.

21. Parkin J and Cohen B: An overview of the immune system. Lancet 357: 1777-1789, 2001.

22. Kiladjian JJ, Giraudier S and Cassinat B: Interferon-alpha for the therapy of myeloproliferative neoplasms: Targeting the malignant clone. Leukemia 30: 776-781, 2016.

23. Cao ZH, Zheng QY, Li GQ, Hu XB, Feng SL, Xu GL and Zhang KQ: STAT1-mediated down-regulation of Bcl-2 expression is involved in IFN- $\gamma /$ TNF- $\alpha$-induced apoptosis in NIT-1 cells. PLoS One 10: e0120921, 2015.

24. Ethiraj P, Veerappan K, Samuel S and Sivapatham S: Inhibitory effects of interferon- $\beta$ on hepatocellular carcinoma HepG2 via Akt/STAT phosphorylation. Fundam Clin Pharmacol 29: 278-285, 2015.

25. Bello C, Vazquez-Blomquist D, Miranda J, Garcia Y, Novoa LI, Palenzuela D and Bello I: Regulation by IFN- $\alpha /$ IFN- $\gamma$ co-formulation (HerberPAG ${ }^{\circledR}$ ) of genes involved in interferonSTAT-pathways and apoptosis in U87MG. Curr Top Med Chem 14: 351-358, 2014.

26. Zídek Z, Jansa P and Kmoníčková E: Activation of respiratory complex II by interferon-gamma and its inhibition by pyrimidine derivatives. Neuro Endocrinol Lett 35 (Suppl 2): 141-148, 2014.

27. Huang G, Chen Y, Lu H and Cao X: Coupling mitochondrial respiratory chain to cell death: An essential role of mitochondrial complex I in the interferon-beta and retinoic acid-induced cancer cell death. Cell Death Differ 14: 327-337, 2007.

28. Kim KB, Eton O, East MJ, Hodges C, Papadopoulos NE, Grimm EA and Bedikian AY: Pilot study of high-dose, concurrent biochemotherapy for advanced melanoma. Cancer 101: 596-603, 2004

29. Henry SC, Schmidt EA, Fessler MB and Taylor GA: Palmitoylation of the immunity related GTPase, Irgm1: Impact on membrane localization and ability to promote mitochondrial fission. PLoS One 9: e95021, 2014.

30. Yang JY, Deng W, Chen Y, Fan W, Baldwin KM, Jope RS, Wallace DC and Wang PH: Impaired translocation and activation of mitochondrial Akt1 mitigated mitochondrial oxidative phosphorylation Complex V activity in diabetic myocardium. J Mol Cell Cardiol 59: 167-175, 2013

31. Tian Z, Miyata K, Kadomatsu T, Horiguchi H, Fukushima H, Tohyama S, Ujihara Y, Okumura T, Yamaguchi S, Zhao J, et al: ANGPTL2 activity in cardiac pathologies accelerates heart failure by perturbing cardiac function and energy metabolism. Nat Commun 7: 13016, 2016.

32. Sansbury BE, Riggs DW, Brainard RE, Salabei JK, Jones SP and Hill BG: Responses of hypertrophied myocytes to reactive species: Implications for glycolysis and electrophile metabolism. Biochem J 435: 519-528, 2011.

33. Zorov DB, Juhaszova M and Sollott SJ: Mitochondrial reactive oxygen species (ROS) and ROS-induced ROS release. Physiol Rev 94: 909-950, 2014.

34. Chin YE, Kitagawa M, Kuida K, Flavell RA and Fu XY: Activation of the STAT signaling pathway can cause expression of caspase 1 and apoptosis. Mol Cell Biol 17: 5328-5337, 1997.

35. Smith CC, Dixon RA, Wynne AM, Theodorou L, Ong SG, Subrayan S, Davidson SM, Hausenloy DJ and Yellon DM: Leptin-induced cardioprotection involves JAK/STAT signaling that may be linked to the mitochondrial permeability transition pore. Am J Physiol Heart Circ Physiol 299: H1265-H1270, 2010.

36. Lemoine S, Zhu L, Legallois D, Massetti M, Manrique A and Hanouz JL: Atorvastatin-induced cardioprotection of human myocardium is mediated by the inhibition of mitochondrial permeability transition pore opening via tumor necrosis factor- $\alpha$ and Janus kinase/signal transducers and activators of transcription pathway. Anesthesiology 118: 1373-1384, 2013.

37. Dvorak K and Dvorak B: Role of interleukin-6 in Barrett's esophagus pathogenesis. World J Gastroenterol 19: 2307-2312, 2013.

38. Santambrogio P, Erba BG, Campanella A, Cozzi A, Causarano V, Cremonesi L, Gallì A, Della Porta MG, Invernizzi R and Levi S: Over-expression of mitochondrial ferritin affects the JAK2/ STAT5 pathway in K562 cells and causes mitochondrial iron accumulation. Haematologica 96: 1424-1432, 2011. 
39. Park SJ1, Lee JH, Kim HY, Choi YH, Park JS, Suh YH, Park SM, Joe EH and Jou I: Astrocytes, but not microglia, rapidly sense $\mathrm{H}_{2} \mathrm{O}_{2}$ via STAT6 phosphorylation, resulting in cyclooxygenase-2 expression and prostaglandin release. J Immunol 188: 5132-5141, 2012.

40. Jesel M,Isch-TreussardC,Poenaru S and Isch F: Electromyography in the study of thyrotoxic myopathies. Rev Electroencephalogr Neurophysiol Clin 3: 183-192, 1973 (In French).

41. Sartor CI, Dziubinski ML, Yu CL, Jove R and Ethier SP: Role of epidermal growth factor receptor and STAT-3 activation in autonomous proliferation of SUM-102PT human breast cancer cells. Cancer Res 57: 978-987, 1997.

42. Sahu RP and Srivastava SK: The role of STAT-3 in the induction of apoptosis in pancreatic cancer cells by benzyl isothiocyanate. J Natl Cancer Inst 101: 176-193, 2009.

43. Thoennissen NH, Iwanski GB, Doan NB, Okamoto R, Lin P, Abbassi S, Song JH, Yin D, Toh M, Xie WD, et al: Cucurbitacin B induces apoptosis by inhibition of the JAK/STAT pathway and potentiates antiproliferative effects of gemcitabine on pancreatic cancer cells. Cancer Res 69: 5876-5884, 2009.
44. Liby K, Voong N, Williams CR, Risingsong R, Royce DB, Honda T, Gribble GW, Sporn MB and Letterio JJ: The synthetic triterpenoid CDDO-Imidazolide suppresses STAT phosphorylation and induces apoptosis in myeloma and lung cancer cells Clin Cancer Res 12: 4288-4293, 2006.

45. Mora LB, Buettner R, Seigne J, Diaz J, Ahmad N, Garcia R, Bowman T, Falcone R, Fairclough R, Cantor A, et al: Constitutive activation of Stat 3 in human prostate tumors and cell lines: Direct inhibition of Stat 3 signaling induces apoptosis of prostate cancer cells. Cancer Res 62: 6659-6666, 2002.

46. Ni Z, Lou W, Leman ES and Gao AC: Inhibition of constitutively activated Stat 3 signaling pathway suppresses growth of prostate cancer cells. Cancer Res 60: 1225-1228, 2000.

47. Burke WM, Jin X, Lin HJ, Huang M, Liu R, Reynolds RK and Lin J: Inhibition of constitutively active Stat 3 suppresses growth of human ovarian and breast cancer cells. Oncogene 20: 7925-7934, 2001.

48. Bromberg J: Stat proteins and oncogenesis. J Clin Invest 109: 1139-1142, 2002. 